\title{
Decoherence of electron spin qubits in Si-based quantum computers
}

\author{
Charles Tahan, Mark Friesen, and Robert Joynt \\ Department of Physics, University of Wisconsin-Madison \\ 1150 University Avenue, Madison, WI 53706
}

(Dated: October 23, 2018)

\begin{abstract}
Direct phonon spin-lattice relaxation of an electron qubit bound by a donor impurity or quantum dot in SiGe heterostructures is investigated. The aim is to evaluate the importance of decoherence from this mechanism in several important solid-state quantum computer designs operating at low temperatures. We calculate the relaxation rate $1 / T_{1}$ as a function of [100] uniaxial strain, temperature, magnetic field, and silicon/germanium content for Si:P bound electrons. The quantum dot potential is much smoother, leading to smaller splittings of the valley degeneracies. We have estimated these splittings in order to obtain upper bounds for the relaxation rate. In general, we find that the relaxation rate is strongly decreased by uniaxial compressive strain in a SiGe-Si-SiGe quantum well, making this strain an important positive design feature. Ge in high concentrations (particularly over 85\%) increases the rate, making Si-rich materials preferable. We conclude that SiGe bound electron qubits must meet certain conditions to minimize decoherence but that spin-phonon relaxation does not rule out the solid-state implementation of error-tolerant quantum computing.
\end{abstract}

PACS numbers: 3.67.Lx,85.35.Gv,72.25.Rb

\section{INTRODUCTION}

The prospect of quantum computing (QC) has caused great excitement in condensed matter physics. If a set of qubits can be maintained in a coherent, controllable many-body state, certain very difficult computational problems become tractable. In particular, successful QC would mean a revolution in the areas of cryptography and data-base searching2. In addition, it would mean a great advance in general technical capabilities, since the control of individual quantum systems and their interactions would represent a new era in nanotechnology.

However, from a practical point of view, a dilemma presents itself immediately. On the one hand, one wishes to control quantum degrees of freedom using external influences, since that is how a quantum algorithm is implemented, and to measure them, since that is the output step. On the other hand, the system must be isolated from the environment, since random perturbations will destroy the quantum coherence that is the whole advantage of QC. This is the isolation-control dilemma, and it leads to a very rough figure-of-merit $F$ for any quantum computer. If we define the decoherence time $\tau$ as the time it takes to lose quantum coherence, and the clock speed $s$ (roughly the inverse of the time to run a logic gate), then the figure of merit is $F=s \tau$ and a practical machine should satisfy $F>10^{4}-10^{5}$ at least. If the clock speed is limited only by standard electronics, then we may be able to achieve $s \approx 10^{9} \mathrm{~Hz}$. This would imply that $\tau=1 \mathrm{~ms}$ is a lower limit for the decoherence time.

The dilemma has not yet been solved, though a number of solutions have been proposed. A particularly attractive solution is to use spin degrees of freedom as qubits. Nuclear spins interact relatively weakly with their environment because the coupling, proportional to the magnetic moment, is small. Yet there has grown up a sophisticated technology (NMR) for the manipulation of the spins, and some rudimentary computations have been performed $\mathbf{B}$. Readout is the main difficulty with this approach, since the field created by a single moment is tiny, and pure states cannot be achieved in the macroscopic samples used.

Electron spins also interact weakly with the environment in some circumstances: relaxation times in excess of $10^{3} \mathrm{~s}$

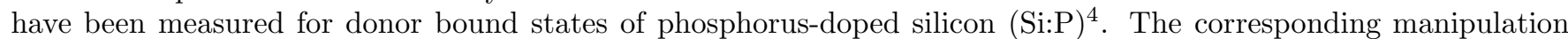
technology (ESR) has also reached a high level of sophistication, but the magnetic moment of the electron exceeds that of the nucleus by 3 orders of magnitude, which presents problems of isolation. Readout should be easier than for nuclei, since detection of single electron charges is certainly possibleE, and spin-dependent detection is not far out of the reach of current technology.

Solid-state implementations of QC are particularly attractive because of the possibility of using existing computer technology to scale small numbers of qubits up to the $10^{5}$ or so that would be needed for nontrivial computations. The first paper to propose using the electron spin in a quantum dot subjected to a strong DC magnetic field was that of Loss and DiVincenzol. Kand proposed employing the nuclear spin in the Si:P system as the qubit. A specific structure consisting of silicon-germanium (SiGe) layers was proposed by Vrijen et al.8. This structure incorporates the idea that the $g$-factor of an electron can be changed by moving it in a Ge concentration gradient, allowing individual electron to be addressed by the external AC field. A different SiGe structure has been proposed by Friesen et al. $\mathrm{E}$. This structure is designed so that the electron number on the dots, and the coupling between the dots, can be carefully 
controlled.

Solid-state implementations must also face the isolation-control dilemma. Decoherence times must exceed the $1 \mathrm{~ms}$ number in the actual physical structures that are needed for the operation of quantum algorithms. In this paper, we examine whether this can be the case for some of the existing proposals based on electron-spin qubits. In the process, we hope to learn something about modifications to these structures that can increase $\tau$. We shall focus on low temperature operation, since, as we shall see, this will probably be necessary in order to obtain sufficiently large $\tau$.

We can build on a large body of work, both theoretical and experimental, from the 1950's and 1960's on ESR in doped semiconductors. In a series of papers, Feher and collaboratorst investigated the relaxation time for the spin of electrons bound on donor sites in lightly doped Si. At sufficiently low temperatures, the relaxation time $T_{1}$ is dominated by single-phonon emission and absorption. In the presence of spin-orbit cpupling (SOC), this can relax the spin, causing decoherence. The theory was worked out by Hasegawa 10 and Roth 11 .

It must of course be recognized that this spin-lattice relaxation time is not necessarily to be identified with the decoherence time. The decoherence time is the shortest time for any process to permanently erase the phase information in the wavefunction. This may mean the phase for a single spin, but it also means that the relative phases of the wavefunctions of different spins must also be preserved, so that processes that cause mutual decoherence must also be taken into account. The actual decoherence time is the minimum of all of these times. A spin relaxation time in excess of $1 \mathrm{~ms}$ is a necessary, not a sufficient, condition for the viability of a solid-state electron-spin QC proposal.

A QC must have precise input as well as an accurate algorithm. Preparation of the spin state is often proposed to be done by thermalization of the spin system at a low temperature. The time to do this actually sets an upper limit on the relaxation time of whatever processes thermalize the spins to the lattice. A limit of perhaps $1-10 s$ is a reasonable requirement.

This paper focuses on $T_{1}$, the time for relaxation of the longitudinal component of the magnetization, by spin-phonon interactions. These processes cause real spin-flip transitions. They occur at random times and thus indubitably cause decoherence. In addition to these processes characterized fully by $T_{1}$, there are processes which introduce random phase changes in the spin wavefunctions. To characterize all such processes by a single "dephasing time" $T_{2}$ will usually not be sufficient for understanding the operation of a multi-qubit system 12 . Difficulties of definition arise, and care must be taken to specify which phase is involved and to what extent it is randomized. For a single spin system there is no ambiguity. The $2 \times 2$ density matrix $\rho_{i j}$ for the qubit with cylindrical symmetry involves only 2 independent parameters $\rho_{11}-\rho_{22}$, and $\rho_{12}$. The time dependence of $\rho_{11}-\rho_{22}$ after a system preparation is exponential with decay constant $T_{1}$ and represents the return of the longitudinal component of the magnetization to its equilibrium value. The decay is due to inelastic transitions of the type calculated in this paper. $\rho_{12}$ on the other hand, is nonzero only if the preparation of the spin state has a transverse component: $S_{x}(t=0) \neq 0$. The decay of this quantity represents the irreversible conversion of this state to an incoherent mixure of "up" and "down" states. Again, this is genuine decoherence of the spin state, since the phase information cannot be recovered. The time dependence of $\rho_{12}$ when the spin is in a strong field has been calculated by Mozyrsky et al 13 using a Markovian approximate master equation. They find that the time scale of the decay due to spin-phonon coupling is very short, of the order of the time for a phonon to cross the electron's wavefunction, which is about $10^{-10} s$. But the decay is incomplete, with $\rho_{12}$ retaining all but $10^{-8}$ of its original value. Their calculation was for Si:P, but a very similar result should hold for the dot case. Decoherence at this level is certainly acceptable for quantum computing. These authors also pointed out that the decay of the remainder of $\rho_{12}$ is due to the spin-flip processes computed in this paper. If $T_{2}$ is defined as the dominant decay time of the off-diagonal density matrix element, then $T_{1}=T_{2}$ for spin-phonon processes.

A quite different source of decoherence is the hyperfine coupling to nuclear spins. The nuclear spins produce an effective random magnetic field on the electrons. Very recent calculations using semiclassical averaging techniquest 4 have obtained a very short relaxation time $T_{\Delta} \approx 1 \mathrm{~ns}$ for GaAs-based dot systems in a strong field. This represents the decay of the transverse magnetization of an ensemble of dots. This is a dephasing time, but not a decoherence time. The electrons spins precess in what is effectively the frozen field of the nuclei. This field is spatially random, and the differential precession of the electron spins leads to the magnetization decay. However, this is not an irreversible loss of the phase information of the collective wavefunction. Spin echo exeriments are a very beautiful demonstration of precisely this point. This "inhomogeneous broadening" presents challenges for the calibration and operation of quantum computers, but does not destroy coherence.

Finally, in any implementation based on electron spin qubits, there will certainly exist small interactions between the spins themselves. The dipole-dipole interaction, for one, cannot be avoided, and there may be indirect spin-spin interactions mediated by the gates. A recentpaper suggests that these interactions set the fundamental time scale $T_{M}$ for Si quantum dot implementations of QC 2 . These interactions do produce experimental broadening of ESR lines in experiments on bulk systems, and this might be taken as decoherence. In our view, however, these interactions do not 
destroy the coherence of a state. The system is the set of all the qubits. During the course of a quantum algorithm they are collectively in a pure state (in principle). Any decoherence that destroys the purity of the state comes from averaging over the unknown states of the environment. The broadening that comes from dipole-dipole interactions comes, in NMR and ESR calculations, from averaging over the states of the system itself, which is not an appropriate method for calculating decoherence. The effect of qubit-qubit interactions that cannot be turned off is to complicate the quantum algorithm. A quantum algorithm is a unitary transformation that must always include the effect of the system Hamiltonian (including dipole-dipole interactions) in addition to external operations. In every case except for very simple ones, this algorithm must first be computed, presumably with the help of a classical computer. This step in QC may be termed "quantum compilation". The issue that qubit-qubit interactions raise is not one of decoherence, but rather whether the determination of the algorithm, the complation step, becomes prohibitively difficult. This could happen for two reasons. One is that the interactions are so poorly known that they cannot be corrected for. It seems likely that quantum error correction can resolve this difficulty. A second and more interesting possibility that the interactions convert the computation of the algorithm itself into a problem that grows exponentially with the size of the system. We regard this as an open question and a deep one, that combines many-body theory with algorithm design and error correction. We note that in NMR implementations the interaction between the qubits also cannot be turned off, but it can be canceled by refocusing 16 .

In this paper, our aim is to evaluate the importance of spin-phonon coupling as a source of decoherence in quantum dot qubits. Fundamentally, the issue is whether the long relaxation times $T_{1}$ observed at low temperatures in bulk Si:P carry over to SiGe dots proposed for QC. In Sec. II we introduce the structures that we are interested in. In Sec. III the physics of the spin-phonon relaxation mechanism is described qualitatively. In Sec. IV calculations and results for pure Si systems are given as a function of the critical design and operating parameters. In Sec. V are found the correponding results for structures containing Ge. Sec. V1 is the conclusion.

\section{THE STRAINED SILICON QUANTUM WELL}

Si-Ge heterostructures are utilized widely in the digital electronics industry, and presently have the shortest switching times of any device. One reason for their success lies in the ability to engineer structures of near perfect purity, with control over thicknesses and interfaces that approaches atomic precision - a technological tour-de-force. An equally key achievement has been the harnessing of strain as a tool to control band offsets in heterostructure devices This paper presepts calculations of spin relaxation for real SiGe structures such as those proposed by Vrijen et al. and Friesen et al. . Accordingly, we have calculated the electron wavefunctions in quantum wells, which is needed as input for these calculations. Details of these calculations are presented in Ref.E, and will not be repeated here. In this section we only describe those aspects of the calculations that are germane to spin relaxation.

Quantum wells are constructed by sandwiching a very thin layer of one material between two others. Electrons can be confined in the quantum well layer when the conduction band offsets produce a potential well. The key to this technology is therefore to understand the band structure of the various layers. In this section we will consider a particular class of wells formed of pure $\mathrm{Si}$, sandwiched between barrier layers of SiGe. We will find that this is optimal from the standpoint of spin coherence. Metallic gates or impurities create zero-dimensional bound states that define a quantum dot. We first review briefly effective mass theory for dots in pure, unstrained Si, then unstrained SiGe, and finally strained Si.

In pure $\mathrm{Si}$, the $\Delta$ conduction band minima occur near the symmetry points $X$, in the directions $\{001\}$. In a perfect Si crystal these minima are six-fold degenerate, the valleys being equivalent. In the dot the electron feels a potential $V_{g}(\vec{r})$ in addition to the atomic potential, which lifts the degeneracy, though the splittings are not large. The spatial variation of $V_{g}(\vec{r})$ is on length scales generally much longer than the lattice spacing. For the moment, we shall assume that the electron is in the ground state of $V_{g}(\vec{r})$ and ignore mixing with any excited states. To the extent that the scale of variation of $V_{g}(\vec{r})$ is much longer than the atomic spacing there are six pearly degenerate ground states. This is referred to as the "valley degeneracy". The wavefunctions can be written ast

$$
\Phi_{n}(\vec{r})=\sum_{j=1}^{6} \alpha_{n}^{(j)} F_{j}(\vec{r}) \phi_{j}(\vec{r}) .
$$

Here, $\phi_{j}$ is a Bloch function of the form

$$
\phi_{j}(\vec{r})=u_{j}(\vec{r}) e^{i \vec{k}_{j} \cdot \vec{r}}
$$

where $\vec{k}_{j}$ are the six $\Delta$ minima $\left\{+k_{0} \hat{x},-k_{0} \hat{x},+k_{0} \hat{y},-k_{0} \hat{y},+k_{0} \hat{z},-k_{0} \hat{z}\right\}$ (we shall always use this ordering), and $u_{j}(\vec{r})$ are periodic functions with the same periodicity as the crystal potential $V_{p}(\vec{r})$. The $F_{ \pm z}$ are envelope functions that 
satisfy the Schrödinger-like equation

$$
\left.\left[-\frac{\hbar^{2}}{2 m_{l}} \frac{\partial^{2}}{\partial z^{2}}-\frac{\hbar^{2}}{2 m_{t}}\left(\frac{\partial^{2}}{\partial x^{2}}+\frac{\partial^{2}}{\partial y^{2}}\right)+V_{g}(\vec{r})\right] F_{ \pm z}(\vec{r})\right)=\left(E-E_{k_{z}}^{(\Delta)}\right) F_{ \pm z}(\vec{r})
$$

and are independently normalized to unity, similar to wavefunctions. Analogous equations can be given for the $\pm \hat{\mathbf{x}}$ and $\pm \hat{\mathbf{y}}$ minima. We see that $F_{x}=F_{-x}, F_{y}=F_{-y}$, and $F_{z}=F_{-z}$, so only three independent envelope functions must be computed. $m_{l}$ and $m_{t}$ are the longitudinal and transverse effective masses associated with the anisotropic conduction band valleys. $E_{k_{z}}^{(\Delta)}$ is the $\Delta$ conduction band edge at $\mathbf{k}_{z}$. The splitting of the degeneracy comes from corrections to this envelope fuction approximation. Different choices of the constants $\alpha_{n}^{(j)}$ determine the six states. Their values will be discussed in Sec. III. This formalism is a good approximation for both dot and impurity bound states, as the valley splitings are much smaller than the energy scales in 3 .

Germanium is completely miscible in $\mathrm{Si}$, forming a random alloy. For variable Ge content $x, \mathrm{Si}_{1-x} \mathrm{Ge}_{x}$ exhibits materials properties that vary gradually over the composition range. The alloy lattice constant, $a_{0}(x)$, follows a linear interpolation between pure Si and Ge, known as Vegard's law, quite accurately for all $x: a_{0}(x)=(1-x) a_{\mathrm{Si}}+x a_{\mathrm{Ge}} 20$. Electronic properties show an abrupt change in behavior near $x \simeq 0.85$, where the Si-like $\Delta$ minima cross over to four-fold degenerate, Ge-like, $L$ minima. In this work we focus on the range $x \lesssim 0.5$, which is strictly Si-like, though we will have some remarks below on Ge-rich structures. Throughout this range, properties such as effective mass and the dielectric constant vary only slightly from pure Si values. For our calculations, the most important parameter is the conduction band edge, $E^{(\Delta)}(x)$, which remains 6 -fold degenerate in the range $x \lesssim 0.5$. The theory of the variation of $E^{(\Delta)}$ with $x$ is not germane to the present work, and we simply quote the empirical result, linear in $x$, which is consistent with Ref.18:

$$
\Delta E^{(\Delta)}(x)=E^{(\Delta)}(x)-E^{(\Delta)}(0) \simeq 0.23 x(\mathrm{eV})
$$

(We note, however, that Ref.19 suggests a slope for $E^{(\Delta)}(x)$ of opposite sign.) The relatively weak variations of the effective mass and the dielectric constant will be ignored here.

We consider thin Si wells in which the Si layer grows pseudomorphically. The in-plane lattice constant, $a_{\|}$, must be the same for all the layers, causing a tetragonal distortion in the strained layer(s). Here we consider the case of strained $\mathrm{Si}$ grown on the (001) surface of relaxed $\mathrm{Si}_{1-x} \mathrm{Ge}_{x}$. The in-plane Si lattice constant depends on $x$ as

$$
a_{\|}(x)=(1-x) a_{\mathrm{Si}}+x a_{\mathrm{Ge}}
$$

Since $a_{\mathrm{Ge}}>a_{\mathrm{Si}}$, the $\mathrm{Si}$ is under tensile strain in the plane. Hence, the out-of-plane Si lattice constant, $a_{\perp}$, is reduced according to continuum elastic theory:

$$
a_{\perp}(x)=a_{\mathrm{Si}}\left[1-2 \frac{c_{12}}{c_{11}} \frac{a_{\|}(x)-a_{\mathrm{Si}}}{a_{\mathrm{Si}}}\right]
$$

where $c_{11}$ and $c_{12}$ are elastic constants for pure Si.

Strain produces shifts of the $\Delta$ band proportional to the strain variables

$$
\varepsilon_{\|}(x)=\frac{a_{\|}(x)-a_{\mathrm{Si}}}{a_{\mathrm{Si}}} \quad \text { and } \quad \varepsilon_{\perp}(x)=\frac{a_{\perp}(x)-a_{\mathrm{Si}}}{a_{\mathrm{Si}}} .
$$

with proportionality constants called the dilational and uniaxial deformation potentials, $\Xi_{d}^{(\Delta)}$ and $\Xi_{u}^{(\Delta)}$, respectively. Because of the anisotropic nature of the strain, the two $\hat{\mathbf{z}}$ minima are shifted down relative to the $\hat{\mathbf{x}}$ and $\hat{\mathbf{y}}$ minima, resulting in a splitting of the $\Delta$ conduction band. The net shifts with respect to the unstrained Si $\Delta$ band are given by 18

$$
\begin{aligned}
& \Delta E^{\left(\Delta_{\perp}\right)}(x)=\left(\Xi_{d}^{(\Delta)}+\frac{1}{3} \Xi_{u}^{(\Delta)}\right)\left(2 \varepsilon_{\|}(x)+\varepsilon_{\perp}(x)\right)+\frac{2}{3} \Xi_{u}^{(\Delta)}\left(\varepsilon_{\perp}(x)-\varepsilon_{\|}(x)\right), \\
& \Delta E^{\left(\Delta_{\|}\right)}(x)=\left(\Xi_{d}^{(\Delta)}+\frac{1}{3} \Xi_{u}^{(\Delta)}\right)\left(2 \varepsilon_{\|}(x)+\varepsilon_{\perp}(x)\right)-\frac{1}{3} \Xi_{u}^{(\Delta)}\left(\varepsilon_{\perp}(x)-\varepsilon_{\|}(x)\right) .
\end{aligned}
$$

The first terms in Eqs. (8) and (9) are hydrostatic strain terms, which lower the conduction edge compared to unstrained Si. The second terms in Eqs. (8) and (9) produce the splitting, associated with uniaxial strain. To perform our calculations, we use the materials parameters given in Table I. However we note that the deformation potentials, particularly $\Xi_{d}^{(\Delta)}$, are very difficult to measure experimentally. Considerable disagreement exists in the literature as 
to the value and even the sign of $\Xi_{d}^{(\Delta)}$ 21. The value given in Table 1 wasreported (but not endorsed!) in Ref 21, and provides energy band variations in general agreement with Refs. 18 and 19 . We arrive at the following strain-induced shifts of the conduction band edge for pure Si:

$$
\Delta E^{\left(\Delta_{\perp}\right)}=-0.86 x \quad \text { and } \quad \Delta E^{\left(\Delta_{\|}\right)}=-0.16 x .
$$

The corresponding shift in the relaxed barrier layers, due to the presence of Ge, was given in Eq. (何). Together, these results describe the conduction band offsets for the quantum well that are used in our simulations.

We now apply our results to two specific quantum well designs of interest for quantum computing. Design (1), shown in the inset of Fig. 1, is a version of that proposed by Vrijen et al.8, in which electrons are trapped on donor ions (usually P), implanted in a semiconductor matrix. In that work, the quantum well is split into Ge-rich and Si-rich regions to facilitate single qubit operations. For simplicity, we consider here a uniform quantum well, formed of pure $\mathrm{Si}$, with a single dopant ion located at the center of the well. In such a device, single qubit operations can be accomplished using a coded qubit scheme22. Design (2), proposed by Friesen et al.9, is shown in Fig. 2. The confinement potential for the electrons is much softer than in design (1). Electrons are trapped vertically by the quantum well, and laterally by the electrostatic potential arising from lithographically patterned, metallic top-gates. Additionally, the quantum dot is tunnel-coupled to a degenerate doped back-gate. The dimensions for both designs are given in the figures.

The wave function of the bound electron is computed in the envelope function formalism, Eq. (3). Couplings between the different valleys are introduced through the perturbation theory described in Sec. IV. This procedure provides the specific values of $\alpha_{g}^{(i)}$ for the ground state, which we use in our calculations.

The abrupt conduction band offsets are handled by matching the ground state wave function $\Phi_{g}(\vec{r})$ and $\partial_{z} \Phi_{g}(\vec{r})$ at the interfaces. (Remember that we have equated effective masses on both sides of interfaces.) Due to linear independence of the Bloch functions, the boundary conditions do not cause mixing of the envelope functions. Solutions of Eq. (3) and the analogous $F_{x}$ equation are obtained, using commercial 3D finite element software.

As will be seen in Sec. V, the key quantity for the computation of $T_{1}$ is $f^{2}$, which describes the probability for the bound electron to be on a Ge atom. Ge is associated with reduced coherence times, by virtue of its large spin-orbit coupling. Referring to Eq. (1), one deduces that this probability may be expressed as

$$
f^{2}=x\left[4\left(\alpha_{g}^{(x)}\right)^{2} \int_{\Omega_{b}} d^{3} r F_{x}^{2}(\mathbf{r})+2\left(\alpha_{g}^{(z)}\right)^{2} \int_{\Omega_{b}} d^{3} r F_{z}^{2}(\mathbf{r})\right],
$$

where $\Omega_{b}$ is the volume outside the quantum well, if the well is pure Si. The subscript $g$ refers to the ground state. The term in square brackets reflects the probability of finding the bound electron in a barrier region, while $x$ gives the probability that the electron is on a Ge site.

Fig. 1 shows the results of our calculations for $f^{2}$ in design (1), as a function of the Ge concentration, $x$, in the $\mathrm{Si}_{1-x} \mathrm{Ge}_{x}$ barriers. For $x>0.02, f^{2}$ decreases with $x$ for two reasons. First, as $x$ increases, the conduction band offset at the quantum well also increases, allowing less of the wavefunction to penetrate the barrier. Second, the spatial extent the electron in the $\hat{\mathbf{z}}$ direction is greater for $F_{x}$ than $F_{z}$, because of the anisotropic effective mass . However, less of $F_{x}$ is mixed into the wavefunction for large $x$, since $\alpha_{x}$ becomes very small. For $x \lesssim 0.02, f^{2}$ drops quickly to zero, due to the absence of Ge in the barriers. In the actual design of Vrijen et al., 8 there is Ge in the active layer. To give an idea of the effect of this, we include an equivalent value of $f^{2}$ for such a structure.

Fig. 2 shows results of the $f^{2}$ calculation for design (2), as a function of the quantum well thickness, $z$. To perform the calculations, we have considered a fixed Ge concentration, $x=0.05$, and taken the limit of large strain, so that $\alpha_{g}^{( \pm x)} \simeq \alpha_{g}^{( \pm y)} \simeq 0$ and $\alpha_{g}^{( \pm z)} \simeq 1 / \sqrt{2}$ for the ground state. As $z$ increases, less of the wavefunction penetrates the barrier regions, causing $f^{2}$ to decrease.

\section{SPIN RELAXATION DUE TO COUPLING TO PHONONS}

In this section we give the method for calculating $T_{1}$, the spin-flip time of a spin qubit in the ground orbital state due to emission or absorption of a phonon, following the logic used by Hasegawa 10 and Roth 11 for bulk Si.

Consider a single impurity with a unit positive charge, such as a phosphorus atom, at the origin. In the absence of central cell corrections, there is a 12-fold degenerate ground state, including spin. This valley degeneracy of the ground state is reduced to two by these corrections, and the splitting between the 2-fold spin-degenerate ground state and the higher states is of order $\Delta E \sim 10 \mathrm{meV}$. We shall discuss the detailed linear combinations ( $\alpha$-values) of the states below, as the coefficients giving the various valley amplitudes play an important role in the calculation of matrix elements. These twelve states may all be thought of as hydrogenic $1 s$ states. The splitting of $1 s$ and $2 s$ is about 
$30 \mathrm{meV}$, larger than the $10 \mathrm{meV}$ valley splittings. Let us now split the 2-fold degenerate ground state by applying a DC magnetic field in the $z$-direction. The transition rates between these states are denoted by $W_{\uparrow \downarrow}$ and $W_{\downarrow \uparrow}$. The relaxation time $T_{1}$ is defined by $1 / T_{1}=W_{\uparrow \downarrow}+W_{\downarrow \uparrow}$.

The transitions are caused by phonons, but there are important approximate symmetries that suppress these transitions. These are: (1) spin rotation symmetry, meaning that the electron spin cannot be flipped by a phonon; this symmetry is broken by SOC; (2) time-reversal symmetry, meaning that one state cannot be changed into its time-reversed partner by emission or absorption of a phonon; this symmetry is broken by the external magnetic field; (3) point group symmetries; these are partially broken when strain is applied.

The spin rotation symmetry would rule out phonon-mediated transitions between the two states entirely if there were no spin-orbit coupling (SOC). This means that the effects of SOC on the wavefunctions, even though these effects are small in relatively low- $Z \mathrm{Si}$, must be taken into account. When we refer to a state as $\uparrow$ or $\downarrow$, these symbols must be taken to refer to the majority spin content of the state, not to a pure spin state. Transition rates are roughly proportional to $(g-2)^{2}$, [more precisely $\left(g_{l}-g_{t}\right)^{2}$, where $g_{t}\left(g_{l}\right)$ is the transverse (longitudinal) $g$-factor, see below for definitions].

The time-reversal symmetry implies that transitions cannot take place directly between Kramers-degenerate states even in the presence of spin-orbit coupling. The direct phonon-mediated transitions between the two states of interest to us are strongly suppressed by this approximate symmetry. It is broken only by the external field $H$. The fastest processes then involve virtual excitation to higher-energy states that are mixed into the ground state by $H$. Hence $1 / T_{1}$ involves a factor $\left(\mu_{B} H / \Delta E\right)^{2}$. There is an additional factor of $H^{2}$ from the phonon density of states, giving an overall rate $1 / T_{1} \sim H^{4}$ in the limit of small $H$.

The point group symmetry is reduced from cubic to tetragonal under strain. This has complex effects that we will explain below.

Before giving actual calculations, we summarize those differences between the electrons in donor impurity states and in an artificial dot that affect $T_{1}$. The most obvious is the single-particle potential that binds the electron. The gate potential is much smoother than the hydrogenic potential of the impurity. This implies that the corrections to the effective mass approximation are much weaker, and $\Delta E$ will be much reduced. It is difficult to compute the energy splittings precisely, but considerations based op the method of Sham and Nakayama 23 give splittings in the range $\sim 0.05-0.1 \mathrm{meV}$ in the structure of Friesen et al.9. This increases the relaxation rate. (In fact a naive estimate of the enhancement is a factor of 400.) On the other hand, the structures we consider have strong lattice strain. This partly lifts the valley degeneracy and also reduces the matrix elements, which decreases the rate. Another aspect of some of the proposed designs is the presence of Ge with its much stronger SOC. This will act to decrease the spin relaxation time.

\section{PURE SI QUANTUM DOTS}

We consider first the case of pure Si under uniaxial strain. The ingredients of the calculation are as follows.

From Sec. II we have the solutions to the Schrödinger equation $\left[\mathcal{H}_{0}+V_{g}(\vec{r})\right] \Phi_{n 0}(\vec{r})=E_{n} \Phi_{n 0}(\vec{r})$. $\mathcal{H}_{0}$ is the unperturbed crystal Hamiltonian without SOC and it has the full space group symmetry. $V_{g}(\vec{r})$ is the gate and/or impurity potential.

To calculate $T_{1}$, we must also include SOC, which we treat as a perturbation: $H_{S O C}=\lambda_{S i} \sum_{\vec{R}} \vec{L}_{\vec{R}} \cdot \vec{S}_{\vec{R}}$. The resulting states $\Phi_{n}(\vec{r})$ are two-fold degenerate because of time-reversal symmetry. Let us denote these states as $\Phi_{n \uparrow}(\vec{r})$ and $\Phi_{n \downarrow}(\vec{r})$. They are not eigenstates of spin, so the arrows denote pseudo-spin. We may define the pseudoup state as that which evolves from the spin-up state as spin-orbit coupling is turned on adiabatically, and similarly for the pseudo-down state. Because of valley and pseudospin degeneracy, there are 2 ground states $\Phi_{g s}(\vec{r})$ and 10 excited states $\Phi_{r s}(\vec{r})$. The 12-fold degeneracy in the effective mass approximation is broken by central-cell corrections in the impurity case and smaller corrections in the quantum dot.

There is also the Zeeman Hamiltonian of the external field $\mathcal{H}_{Z}=\mu_{B} \vec{B} \cdot(\vec{L}+2 \vec{S})$. In the field $\Phi_{n \uparrow}(\vec{r})$ and $\Phi_{n \downarrow}(\vec{r})$ are no longer degenerate. Note that the energy splitting may depend on the direction of $\vec{B}$.

Finally, we have the electron-phonon coupling Hamiltonian $\mathcal{H}_{e p}$. A phonon represents a time-dependent perturbation. This will create transitions whose rate is given by the Fermi golden rule. We are interested in the transitions between $\Phi_{g \uparrow}(\vec{r})$ and $\Phi_{g \downarrow}(\vec{r})$. However, $\left\langle\Phi_{g \uparrow}(\vec{r})\left|\mathcal{H}_{e p}\right| \Phi_{g \downarrow}(\vec{r})\right\rangle=0$ in the absence of the external field. Thus we need to calculate in next order in perturbation theory using an effective Hamiltonian

$$
\mathcal{H}^{\prime}=\sum_{r s} \frac{1}{E_{g}-E_{r}}\left\{\left[\mathcal{H}_{Z}\left|\Phi_{r s}(\vec{r})\right\rangle\left\langle\Phi_{r s}(\vec{r})\right| \mathcal{H}_{e p}\right]+\left[\mathcal{H}_{e p}\left|\Phi_{r s}(\vec{r})\right\rangle\left\langle\Phi_{r s}(\vec{r})\right| \mathcal{H}_{Z}\right]\right\}
$$

Here $r$ runs over the excited states, $r=2, . ., 6 . \quad s=\uparrow, \downarrow$. 
The relaxation time is given by $1 / T_{1}=W_{\uparrow \downarrow}+W_{\downarrow \uparrow}$, where $W_{\downarrow \uparrow}=(2 \pi / \hbar) \times \sum_{\vec{q} \lambda}\left|\left\langle\Phi_{g \uparrow}(\vec{r})\left|\mathcal{H}^{\prime}\right| \Phi_{g \downarrow}(\vec{r})\right\rangle\right|^{2} \delta\left(E_{g \downarrow}-\right.$ $\left.E_{g \uparrow}-\hbar \omega_{\vec{q} \lambda}\right) \quad\left[1+n\left(\omega_{\vec{q} \lambda}\right)\right]$ is the rate for transitions from the higher-energy (pseudo-spin down) state to the lowerenergy (pseudo-spin up) state and $W_{\uparrow \downarrow}=(2 \pi / \hbar) \sum_{\vec{q} \lambda}\left|\left\langle\Phi_{g \downarrow}(\vec{r})\left|\mathcal{H}^{\prime}\right| \Phi_{g \uparrow}(\vec{r})\right\rangle\right|^{2} \delta\left(E_{g \downarrow}-E_{g \uparrow}-\hbar \omega_{\vec{q} \lambda}\right) n\left(\omega_{\vec{q} \lambda}\right)$ is the rate for transitions from the lower-energy (pseudo-spin up) state to the higher-energy (pseudo-spin down) state where the sum is over phonon modes $\vec{q} \lambda$ with energies $\omega_{\vec{q} \lambda}$. A thermodynamic average over the lattice states has been taken. It yields the Bose occupation factors $n\left(\omega_{\vec{q} \lambda}\right)$ for the phonons.

The matrix elements of $\mathcal{H}^{\prime}$ are computed as follows.

The expectation value of the external field $H_{Z}$ can be written as

$$
\begin{aligned}
\left\langle\Phi_{n s}(\vec{r})\left|H_{Z}\right| \Phi_{n^{\prime} s^{\prime}}(\vec{r})\right\rangle & =\sum_{i=1}^{6} \alpha_{n}^{(i)} \sum_{j=1}^{6} \alpha_{n^{\prime}}^{(j)} \mu_{B} \vec{B} \cdot g^{(i)} \cdot \vec{\sigma}_{s, s^{\prime}} \delta_{i j} \\
& =\mu_{B} \vec{B} \cdot\left[\sum_{i=1}^{6} \alpha_{n}^{(i)} \alpha_{n^{\prime}}^{(i)} g^{(i)}\right] \cdot \vec{\sigma}_{s, s^{\prime}} \\
& \equiv \mu_{B} \vec{B} \cdot \mathbf{D}_{n n^{\prime}} \cdot \vec{\sigma}_{s, s^{\prime}}
\end{aligned}
$$

and the tensor $g^{(i)}$ is the effective $g$ factor at the $i$ th valley. This equation defines the tensor $\mathbf{D}_{n n^{\prime}}$ that characterizes the coupling of the various states by the external field. The principal axes of the $g$ tensor are the same as that of the effective mass tensor at the $i$ th valley. It has the form

$$
g^{( \pm x)}=\left(\begin{array}{ccc}
g_{l} & 0 & 0 \\
0 & g_{t} & 0 \\
0 & 0 & g_{t}
\end{array}\right), g^{( \pm y)}=\left(\begin{array}{ccc}
g_{t} & 0 & 0 \\
0 & g_{l} & 0 \\
0 & 0 & g_{t}
\end{array}\right), g^{( \pm z)}=\left(\begin{array}{ccc}
g_{t} & 0 & 0 \\
0 & g_{t} & 0 \\
0 & 0 & g_{l}
\end{array}\right)
$$

There are only two independent constants.

A simple example of the diagonal part of the $\mathbf{D}$ tensor is that for the ground state of an impurity in the unstrained lattice when the central cell corrections are included. Then we have $\alpha_{g}^{(i)}=1 / \sqrt{6}$ and $\mathbf{D}_{g g}$ is proportional to the unit matrix:

$$
\left\langle\Phi_{g s}(\vec{r})\left|H_{z}\right| \Phi_{g s^{\prime}}(\vec{r})\right\rangle=g_{g} \mu_{B} \vec{B} \cdot \vec{\sigma}_{s s^{\prime}}
$$

with

$$
g_{g}=\frac{2}{3} g_{t}+\frac{1}{3} g_{l}
$$

The matrix elements between ground and excited states have the form

$$
\left\langle\Phi_{g s}(\vec{r})\left|H_{Z}\right| \Phi_{r s^{\prime}}(\vec{r})\right\rangle=g^{\prime} \mu_{B} \vec{B} \cdot \mathbf{D}_{g r} \cdot \vec{\sigma}_{s s^{\prime}},
$$

with

$$
g^{\prime}=\frac{1}{3}\left(g_{l}-g_{t}\right)
$$

and the tensor $\mathbf{D}_{r}$ is defined by

$$
\mathbf{D}_{g r}=3 \sum_{i=1}^{6} \alpha_{g}^{(i)} \alpha_{r}^{(i)} \widehat{k}^{(i)} \widehat{k}^{(i)}
$$

where $\widehat{k}^{(i)}$ is the "local" anisotropy axis. If the original $g$ were isotropic, then $g^{\prime}=0$ and there would be no coupling between different states and no spin relaxation.

If the lattice is strained, then the $\alpha$ coefficients become strain-dependent and the general expression for $\mathbf{D}$ from Eq. 13 must be used. Uniaxial strain lifts the degeneracy of the valleys. We include this effect in the Hamiltonian and it determines the proper combinations of the $\alpha_{n}^{(i)}$ defined in Eq. $\mathbb{1}$. These then feed into $\mathbf{D}_{g r}$. As a function of strain the $\alpha_{n}^{(g)}$ for the ground state cross over from the completely symmetric combination $\alpha_{g}^{(i)}=1 / \sqrt{6}$ to the combination $\alpha_{g}^{( \pm x)}=\alpha_{(g)}^{ \pm y}=0, \alpha_{(g)}^{ \pm z}=1 / \sqrt{2}$ in the limit of large strain.t. 
The phonons involved are just the acoustic ones, one longitudinal and two transverse - these are the only ones with low enough energy to play a role in relaxing the spins. The matrix elements of the electron-phonon interaction are only nonzero within one valley and for a single phonon mode they are conventionally parametrized as:

$$
\left\langle\Psi_{c \vec{k} s}^{(i)}(\vec{r})\left|H_{e p}^{(\vec{q} \lambda)}\right| \Psi_{c \vec{k}^{\prime} s}^{(i)}(\vec{r})\right\rangle=i b_{\vec{q} \lambda} \widehat{e}_{\lambda}(\vec{q}) \cdot\left(\Xi_{d} 1+\Xi_{u} \widehat{k}^{(i)} \widehat{k}^{(i)}\right) \cdot \vec{q}+\text { h.c. }
$$

near the $i$ th valley, where $\vec{q}=\vec{k}-\vec{k}^{\prime}$ and $\widehat{e}_{\lambda}$ is the polarization vector. $b_{\vec{q} \lambda}$ destroys a phonon with wavevector $\vec{q}$ and polarization $\lambda$. Once again, we see that the interaction can be characterized by just 2 parameters, in this case $\Xi_{d}$ and $\Xi_{u}$, as already defined in Eq. 7. Performing the integration over the envelope function at wavector $\vec{q}$ now gives

$$
\begin{aligned}
& \left\langle\Phi_{g s}(\vec{r})\left|H_{e p}^{(\vec{q} \lambda)}\right| \Phi_{g s^{\prime}}(\vec{r})\right\rangle_{\vec{q}}=\left(\Xi_{d}+\frac{1}{3} \Xi_{u}\right) A(\vec{q})\left(b_{\vec{q} \lambda}+b_{\vec{q} \lambda}^{*}\right) \delta_{s, s^{\prime}} \\
& \left\langle\Phi_{g s}(\vec{r})\left|H_{e p}^{(\vec{q} \lambda)}\right| \Phi_{r s^{\prime}}(\vec{r})\right\rangle_{\vec{q}}=\frac{1}{3} \Xi_{u} A(\vec{q})\left(i \widehat{e}_{\lambda}(\vec{q}) \cdot \mathbf{D}_{g r} \cdot \vec{q} b_{\vec{q} \lambda}-i \widehat{e}_{\lambda}^{*}(\vec{q}) \cdot \mathbf{D}_{g r} \cdot \vec{q} b_{\vec{q} \lambda}^{*}\right) \delta_{s, s^{\prime}}
\end{aligned}
$$

where

$$
A^{(i)}(\vec{q})=\sum_{\vec{k}} F^{(i) *}(\vec{k}+\vec{q}) F^{(i)}(\vec{k})=\int d^{3} r F^{2}(r) e^{i \vec{q} \cdot \vec{r}}
$$

Thus the electron-phonon interaction involves a form factor for the bound states. Since $F$ is normalized, we have $A^{(i)}(\vec{q}) \approx 1$ when the wavelength of the phonon is much longer than the spatial extent $a^{*}$ of the bound state: $q a^{*}<<1$. The calculations of II indicate that this is the case. It is also independent of $(i)$.

In the golden rule calculation, the energy denoninator $\left(E_{g}-E_{r}\right)^{-2}$ will suppress contributions from the excited states of $V_{g}$. Thus we will keep only states that are split off from the ground state by corrections to the effective mass approximation. This approach works very well in Si:P and should be even better for the quantum dot.

This produces the golden-rule transition rate

$$
\begin{aligned}
W_{\uparrow \downarrow}= & \frac{2 \pi}{\hbar}\left[\frac{1}{3} \Xi_{u} g^{\prime} \mu_{B}\right]^{2} \sum_{\vec{q} \lambda} A^{2}(\vec{q}) \delta\left(E_{g \uparrow}-E_{g \downarrow}-\hbar \omega_{\vec{q} \lambda}\right)\left\langle a_{\vec{q} \lambda} a_{\vec{q} \lambda}^{*}\right\rangle \times \\
& \left|\sum_{r=2}^{6} \frac{\vec{B} \cdot \mathbf{D}_{g r} \cdot \vec{\sigma}_{\uparrow \downarrow} \widehat{e}_{\lambda}(\vec{q}) \cdot \mathbf{D}_{g r} \cdot \vec{q} \delta_{s s^{\prime}}}{E_{g}-E_{r}}\right|^{2} .
\end{aligned}
$$

We approximate the phonon dispersion as $\omega_{\vec{q} \lambda}=v_{\lambda} q$. Setting $A=1$, performing the integral over the magnitude of $\vec{q}$, and repeating the calculation for $W_{\downarrow \uparrow}$, we obtain a total spin relaxation time

$$
\begin{aligned}
\frac{1}{T_{s}}= & W_{\uparrow \downarrow}+W_{\downarrow \uparrow}=\frac{1}{8 \pi^{2} \rho \hbar^{4}}\left(\frac{g^{\prime} \mu_{B} B \Xi_{u}}{3}\right)^{2}\left[2 n\left(g \mu_{B} B\right)+1\right] g_{g}^{3} \mu_{B}^{3} B^{3} \times \\
& \sum_{\lambda=1}^{3} \frac{1}{v_{\lambda}^{5}} \int_{0}^{2 \pi} d \phi^{\prime} \int_{0}^{\pi} \sin \theta^{\prime} d \theta^{\prime}\left|\sum_{r=2}^{6}\left[\vec{B}(\theta, \phi) \cdot \mathbf{D}_{g r} \cdot \vec{\sigma}_{\uparrow \downarrow}\right]\left[\frac{\widehat{e}_{\lambda}(\vec{q}) \cdot \mathbf{D}_{g r} \cdot \widehat{q}}{E_{g}-E_{r}}\left(\theta^{\prime}, \phi^{\prime}\right)\right]\right|^{2} .
\end{aligned}
$$

Here $(\theta, \phi)$ are the polar axes of the direction of $\vec{B}$ measured from the [100] direction of the crystal. $\rho$ is the mass density. This is our basic result, in a form very similar to that given by Hasegawa 10 .

In Fig. 3 the effect of strain on the lowest energy levels is shown. Here the zero-strain splittings are those of a $\mathrm{P}$ impurity. A similar plot was given in Ref.4, but at that time the correct zero-strain splittings were not known. In the dot, the energy splittings for the unstrained case would be two to three orders of magnitude smaller (see below for a discussion).

In Fig. 4, we show the effect of strain on the relaxation time of an electron in a $\mathrm{P}$ impurity potential. There are two effects: the overall increase of the energy denominators and the change of the ground state to a less symmetric valley weighting. This leads to a non-monotonic dependence of $T_{1}$ on strain, but in the region of interest, the effect of strain is to greatly increase $T_{1}$, since most proposed structures have $s<-1$. At large strain, only one energy denominator remains small, that between the ground state, symmetric in the $\pm z$ valleys, and the first excited state, antisymmetric in the $\pm z$ valleys. The overlap matrix $\mathbf{D}$ is very small between these two state. This reduction of the matrix element is the dominant effect. 
Also of interest is the dependence of $T_{1}$ on the angle of the external field, since this may serve as a diagnostic tool in experiments to verify that the relaxation process is really due to spin-phonon coupling. As a function of strain, this dependence becomes highly anisotropic, as seen in Fig. 5. This is due to the elimination of all but the $\pm z$ valleys from the problem at high strain. We note that the limiting value $T_{1} \rightarrow \infty$ when the external field is along a crystal axis is cut off by intervalley scattering effects not included in the present calculation 1 .

The change in the confining potential reduces the corrections to the effective mass approximation, as discussed in Sec. III which in turn reduces the energy denominators, leading to a decrease in $T_{1}$. In the Friesen et al. structure, we have estimated the splitttings $E_{r}-E_{g}$ using the method of Sham and Nakayama23, and they range from 0.05 to $0.1 \mathrm{meV}$, depending on the gate voltages that produce the potential. This may be incorporated into the calculation of the $\alpha_{n}^{(i)}$ by introducing a variable coupling $\Delta_{c}$ that mixes the $\pm x$ and $\pm y$ valley wavefunctions with the $\pm z$ valley wavefunctions. For a precise definition of $\Delta_{c}$, see Ref.4. Approximately, however, $\Delta_{c} \sim 0.2\left(E_{r}-E_{g}\right)<10^{-4} \mathrm{eV}$. The results of the calculations are shown in Fig. 6. The graphs show the absolutely crucial role that strain plays in the determination of $T_{1}$. Because of the small energy denominators in the dot, the rate is extremely fast at small $\Delta_{c}$ for the unstrained case: $1 / T_{1} \sim \Delta_{c}^{-2}$.

We also point out that in stuctures where $T_{1}$ appears to be too long for efficient preparation of the spins (as discussed in Sec. I) the deficiency can be made up by increasing the temperature $T$. As seen in Fig. 7, $T_{1}$ decreases very rapidly as $T$ is increased. Actually, this calculation even underestimates the decrease, since multiphonon processes begin to contribute at about $3 \mathrm{~K}$. However, it must be borne in mind that the temperature must be small enough that the initial state is essentially pure (all spins up, for example). A more practical method of decreasing $T_{1}$ if desired, would be to increase the magnetic field.

\section{STRUCTURES CONTAINING GE}

The effect of alloying with Ge is to increase the SOC and hence to increase $|g-2|$. First principles calculations of Ge impurities in Si have shown that there is little effect on the states near the bottom of the conduction band, though this is not necessarily the case for higher energy states in the band25. This is in accord with the isoelectronic character of the atoms in the alloy. It is then reasonable to employ the virtual crystal approximation (VCA). The approximation should be quantitatively accurate for small, (say $<10 \%$ ) concentrations of Ge, but may be taken as a good qualitative guide also to higher concentrations. The Bloch functions in the absence of SOC satisfy

$$
H_{0} \Psi_{n \vec{k} s}=E_{n \vec{k}} \Psi_{n \vec{k} s}
$$

where

$$
\Psi_{n \vec{k} s}=\frac{1}{\sqrt{N_{c}}} \sum_{j=1}^{N_{c}} \sum_{l=1}^{2} \exp \left(i \vec{k} \cdot r_{j l}\right) \sum_{m=0}^{3} a_{l m}(n \vec{k}) \phi_{m s}\left(\vec{r}-r_{j l}\right)
$$

where $N_{c}$ is the number of unit cells, $j$ labels the unit cells, $l$ labels the two positions in the unit cell, $m$ labels the 4 atomic states, $\phi_{m s}$ are the atomic orbitals, and the coefficients $a_{l m}(n \vec{k})$ give the proper linear combination of atomic orbitals (LCAO's) for the state at momentum $\vec{k}$ and band $n$. The normalization condition is

$$
\sum_{l m}\left|a_{l m}(n \vec{k})\right|^{2}=1
$$

In the VCA, the $a_{l m}(n \vec{k})$ are the same for the $\mathrm{Si}$ and Ge sites. The SOC Hamiltonian for the alloy is written as

$$
H_{S O C}=\sum_{\vec{R}} \lambda_{\vec{R}} \vec{L}_{\vec{R}} \cdot \vec{S}_{\vec{R}}
$$

where $\lambda_{\vec{R}}$ is the SOC strength for $\mathrm{Si}(\mathrm{Ge})$ when $\vec{R}$ is a $\mathrm{Si}(\mathrm{Ge})$ site. The energy shift of an electron in the external field $\vec{B}=\left(B_{x}, B_{y}, B_{z}\right)$ can be written as

$$
\Delta E_{n \vec{k} s}(\vec{B})=-\mu_{B} \sum_{i=x, y, z} g_{i}(n \vec{k}) B_{i} s,
$$


TABLE I: Materials parameters used in this work.

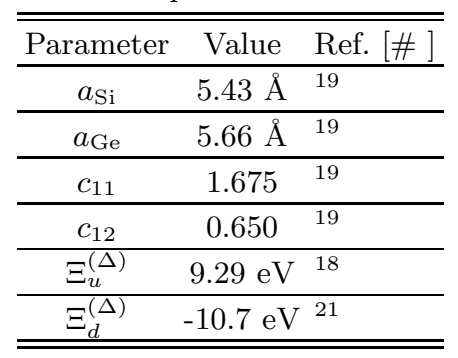

where

$$
g_{i}(n \vec{k})=2-2 \hbar^{2}\left(\sum_{\vec{R}} \lambda_{\vec{R}} / N_{c}\right) \sum_{n^{\prime} \neq n, l, m, m^{\prime}}\left|a_{l m}(n \vec{k})\right|^{2}\left|a_{l m^{\prime}}\left(n^{\prime} \vec{k}\right)\right|^{2}\left(E_{n \vec{k}}-E_{n^{\prime} \vec{k}}\right)^{-1}\left(\varepsilon_{i m m^{\prime}}\right)^{2}
$$

and $\varepsilon_{i m m^{\prime}}$ is the completely antisymmetric symbol with $\varepsilon_{123}=1$.

Our approximation now consists in regarding the dependence on the Ge concentration as coming only in the term $\left(\sum_{\vec{R}} \lambda_{\vec{R}} / N_{c}\right)$. Hence any component of the $g$-tensor is proportional to a weighted average of $\lambda_{S i}$ and $\lambda_{G e}$. In particular, consider the conduction band minimum at $\vec{k}=\left(0,0, k_{0}\right)$ in the compound $\mathrm{Si}_{1-x} \mathrm{Ge}_{x}$. Then we have $g_{z}(x)-2=\left[g_{z}(0)-2\right]\left[(1-x)+x\left(\lambda_{G e} / \lambda_{S i}\right)\right]$, where $g_{z}(0)$ is the value for pure silicon. The numbers, known from atomic physics, are $\lambda_{G e} / \lambda_{S i}=0.295 / 0.044=6.71$, so $g_{z}(x)-2=\left[g_{z}(0)-2\right][(1-x)+6.71 x]$. Similarly, we have $g_{y}(x)-2=\left[g_{y}(0)-2\right][(1-x)+6.71 x]$, etc. Only the overall scale of $g$ changes, not the anisotropy in the VCA.

The presence of Ge in the lattice breaks the translation symmetry, an effect that is neglected in the VCA. Taking this into account would lead to a $g$-factor that is averaged over momentum space rather than one that is evaluated only at the Si conduction band minima. If we take the L-minimum of pure Ge as an example, we would expect that the corrections to $|g-2|$ would be greater if there is some averaging over momentum space. Thus a calculation going beyond the VCA would give corrections that would reduce $T_{1}$.

We show the results for differing Ge concentrations in the active region of the well in Fig. 8. In the Ge-poor regime $x<0.85$, the relaxation rate $1 / T_{1}$ decreases fairly slowly. Thus designs with a Si-rich well will not suffer from very fast spin relaxation on the minority Ge sites.

By contrast, if we cross the critical point around $x=0.85$ where the conduction band minima switch positions, then there is a very rapid decrease of $T_{1}$. This is due to the much greater g-factor anisotropy of the [111] minima: $g^{\prime} \approx-0.4$, (as compared to $g^{\prime}<0.001$ in $\mathrm{Si}$ ). The results for the Ge-rich region are much simpler than in the Si-rich region. Uniaxial strain does not affect the relative valley energies or the matrix elements parametrized by $\mathbf{D}$. The only effect of going to the dot from the impurity is to reduce corrections to the effective mass approximation, which will strongly decrease the relaxation time. Indeed, if we take the impurity result for Ge:P from Ref.10, which is $T_{1}=2.3 \times 10^{-3} \mathrm{~s}$ and estimate the decrease in $\Delta E$ as about a factor of 100 , then we obtain $T_{1} \sim 10^{-6}$ to $10^{-7} s$, which is vastly shorter than that of Si-rich structures. The physics is the same as that which governs the divergence of the rate in Fig. 6 for the unstrained case,namely the much smaller energy denominators.

To make contact with Sec. II note that if there is Ge in the barrier regions, as is the case in most SiGe designs, then we will have an envelope function that weights $\mathrm{Si}$ and Ge differently, then $x$ in the VCA formulas must be replaced by $f^{2}=\sum_{\vec{R}}\left|f_{\vec{R}}\right|^{2}$, where $\left|f_{\vec{R}}\right|^{2}$ is the amplitude that the electron is on a Ge site and the sum runs only over Ge sites, as described in Sec. II. This is the product of the Ge concentration in the layer, times the probability that the electron is on the layer, summed over layers. Referring to Figs. 1 and 2, we see that $f^{2}$ is always less than $10^{-3}$ for structures that have pure $\mathrm{Si}$ active layers and Ge in the barrier layers. The effect on $T_{1}$ is small so these structures are "phonon-safe".

\section{CONCLUSION}

Decoherence due to spin relaxation by emission and absorption of single phonons does not pose a major obstacle to quantum computation in quantum dot SiGe heterostructures using spin qubits, if these qubits are properly designed. This spin relaxation mechanism is the dominant one in the case of electrons in donor bound states, such as in Si:P.

In order that this source of decoherence be kept under control, however, certain conditions must be satisfied in the design of the structure. Unstrained SiGe alloys generally have relatively short spin relaxation times due to valley 
degeneracy. This degeneracy produces strong spin mixing in the Kramers-degenerate ground states of the quantum dot once the field is turned on. This in turn increases the electron-phonon matrix element that connects the two states and decreases the relaxation time. This effect, fundamentally due to spin-orbit coupling, is stronger in the quantum dot than in the impurity state. The corrections to effective-mass theory, stronger for the impurity potential, tend to lift the degeneracy.

The crucial role that strain plays, in Si-rich structures, is to lift the valley degeneracy. In this respect compressive uniaxial strain is the best, since the residual degeneracy is reduced to two valleys. The wavefunction for the ground state is then symmetric in the valley index, and the first excited state, the only one with a small energy denominator, is antisymmetric in this index. This fact strongly suppresses the spin mixing effect of the external field. Thus a workable design should include uniaxial strain that exceeds a critical value. This value is small enough that there will be sufficient strain in most practical structures in which the active layer is Si rich and sandwiched between relatively Ge-rich layers. The active layer must be thin enough to avoid dislocation-mediated relaxation.

Spin-orbit coupling is stronger in Ge than in Si due to the higher atomic number. This suggests that Si-rich structures are to be preferred, and the calculations bear this out. The relaxation time decreases with increasing $x$ in a $\mathrm{Si}_{1-x} \mathrm{Ge}_{x}$ alloy. In fact, if $x$ increases beyond $x=0.85$, decoherence becomes very strong, and quantum dots in these Ge-rich structures, such as those proposed by Vrijen et al 8 , may well run into difficulties for this reason. A key point for these structures is that uniaxial stress does not lift the valley degeneracy, since the valleys have moved off the crystal axes. Strain-induced suppression of spin relaxation cannot occur.

Beyond design issues, fabrication quality is also important. Even a small concentration of magnetic impurities will negatively impact the spin relaxation. In modern semiconductor technology, however, concentrations of magnetic impurities much less than $0.1 \mathrm{ppm}$ are routine. The impact of lattice imperfections is less clear. These will generally act to lower the symmetry of the system, and to lessen the accuracy of the effective-mass approximation. As we have seen in the context of the impurity calculations, these effects generally increase the spin relaxation time because of reduced degeneracy. On the other hand, localized bound states can form at such imperfections. If this results in free electron spins, it could have seriously negative effects on the relaxation time. Similar effects would result from lattice defects that produce localized phonon modes.

Finally, it appears that quantum dot designs will need rather low temperatures in order to operate. The impurity experiments clearly indicate that multi-phonon relaxation increase rapidly with temperature 26, with a crossover to this regime at about $3 \mathrm{~K}$. We speculate that this crossover temperature is pushed up when the effects of strain are included, but we have not yet done the requisite calculations.

We would like to acknowledge useful discussions with our collaborators at the University of Wisconsin-Madison and with Xuedong Hu. This work was supported by the National Science Foundation under the Materials Theory Program, Grant No. DMR-0081039, the Materials Research Science and Engineering Center Program, Grant No. DMR-0079983, and the Army Research Office.

1 P. W. Shor, in Proc. 35th Annu. Symp. on the Foundations of Computer Science, S. Goldwasser, Ed. (IEEE Computer Society Press, Los Alamitos, CA, 1994), pp. 124-134.

2 L. K. Grover, Phys. Rev. Lett. 79, 325 (1997).

3 L. M. K. Vandersypen, et al., Nature 414, 883 (2001).

${ }^{4}$ G. Feher, Phys. Rev. 114, 1219 (1959); G. Feher and E. Gere, 114, 1245 (1959); D. K. Wilson and G. Feher, 124, 1068 (1961).

5 see, e.g., M.A. Kastner, Rev. Mod. Phys. 64, 849 (1991).

6 D. Loss and D. P. DiVincenzo, Phys. Rev. A 57, 120 (1998).

7 B. Kane, Nature 393, 133 (1998).

8 R. Vrijen, E. Yablonovitch, K. Wang, H. Jiang, A. Balandin, V. Roychowdhury, T. Mor, D. DiVencenzo, Phys. Rev. A 62, 012306 (2000).

9 M. Friesen, P. Rugheimer, D. E. Savage, M. G. Lagally, D. W. van der Weide, R. Joynt, M. A. Eriksson, cond-mat/0204035.

10 H. Hasegawa, Phys. Rev. 118, 1523 (1960).

11 L.M. Roth, Phys. Rev. 118, 1534 (1960).

12 X. Hu, R. de Sousa, S. Das Sarma, cond-mat/0108339.

13 D. Mozyrsky, Sh. Kogan, G. P. Berman, cond-mat/0112135.

14 I.A. Merkulov, A.L. Efros, and M. Rosen, cond-mat/0202271.

15 R. de Sousa and S. Das Sarma, cond-mat 0203101.

16 I. Chuang, in Introduction to Quantum Computation and Quantum Information, ed H.-K. Lo, S. Popescu, and T. Spiller (World Scientific, SIngapore, 1998), pp. 320-323.

17 W. Kohn, in Solid State Physics 5, ed. by F. Seitz and D. Turnbull (Academic Press, New York, 1957).

18 M. M. Rieger and P. Vogl, Phys. Rev. B 48, 14276 (1993). 
${ }^{19}$ F. Schäffler, Semicond. Sci. Technol. 12, 1515 (1997).

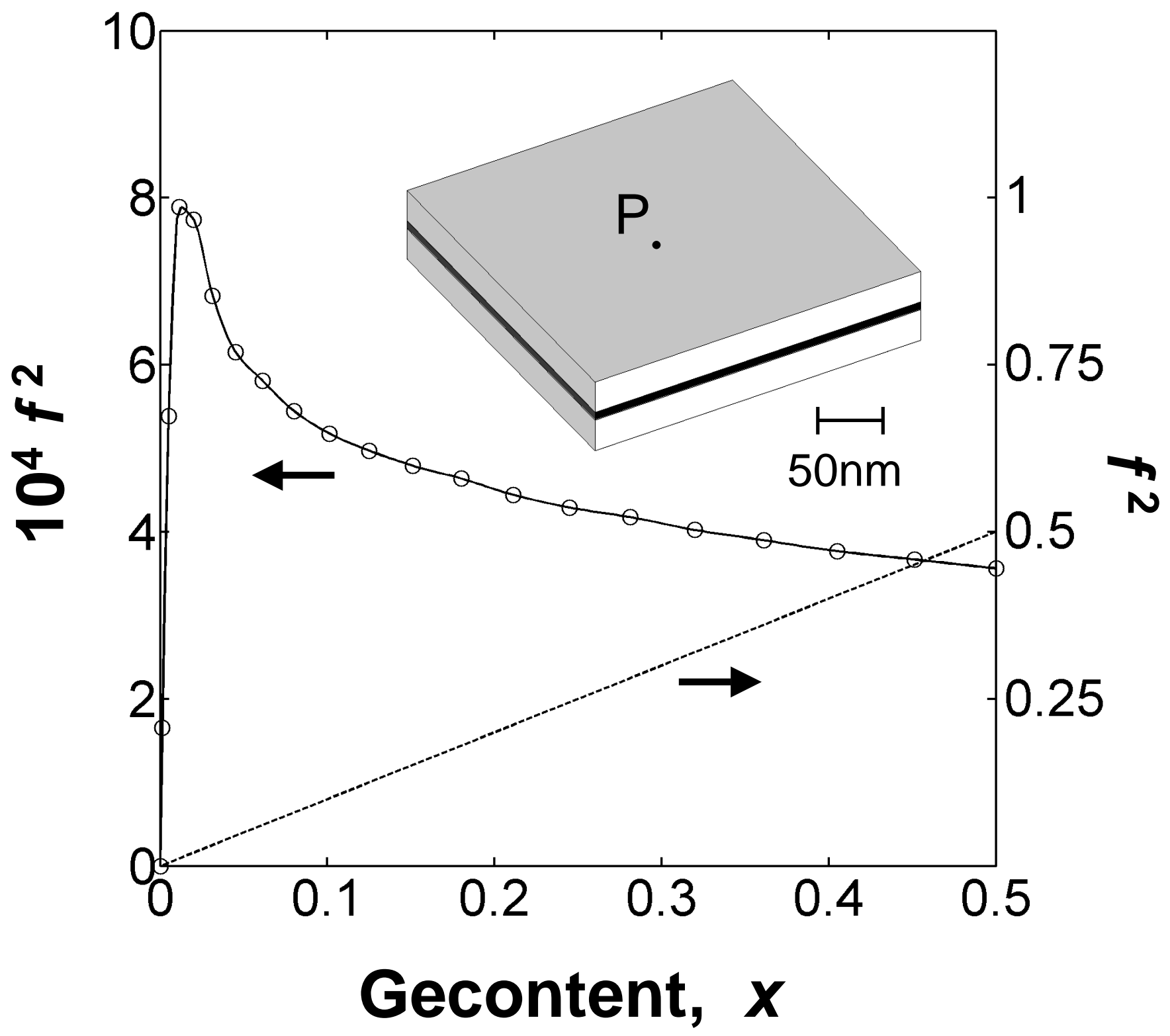

FIG. 1: Probability, $f^{2}$, for finding a donor-bound electron at a Ge site, as a function of Ge content, $x$. The simulated structure, design (1), is shown in the inset. A strained Si quantum well of thickness $6 \mathrm{~nm}$ is sandwiched between relaxed $\mathrm{Si}_{1-x} \mathrm{Ge}_{x}$ barrier regions of thickness $20 \mathrm{~nm}$. The electron is bound to a $\mathrm{P}^{1+}$ ion embedded in the center of the quantum well. 


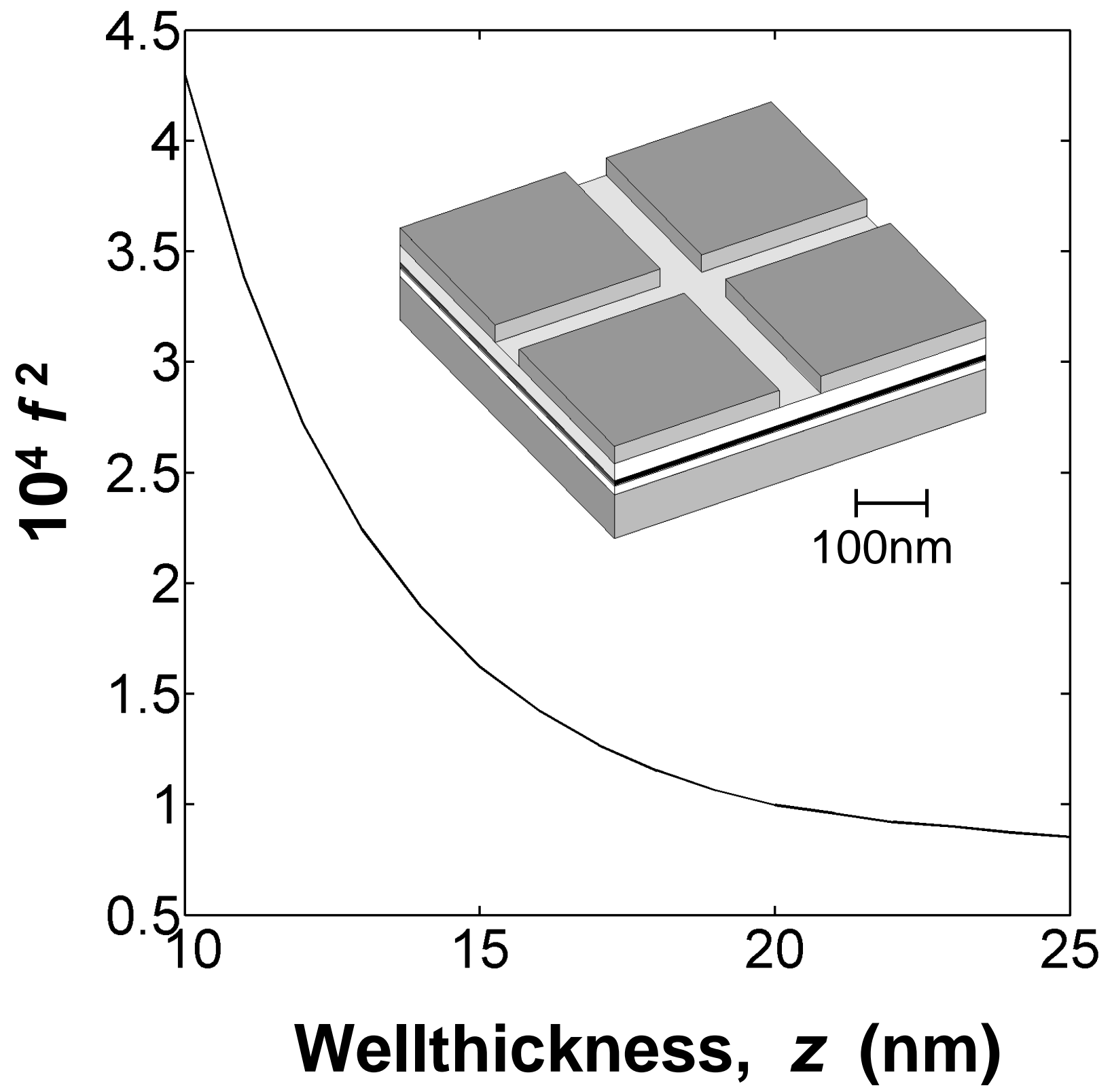

FIG. 2: Probability, $f^{2}$, for finding an electrostatically bound electron at a Ge site, as a function of quantum well thickness, $z$. The inset shows the heterostructure layers for design (2), beginning at bottom: a thick, doped semiconductor back-gate, a relaxed $\mathrm{Si}_{1-x} \mathrm{Ge}_{x}$ barrier layer, a strained $\mathrm{Si}$ quantum well, a thick, relaxed $\mathrm{Si}_{1-x} \mathrm{Ge}_{x}$ barrier layer, and lithographically patterned metallic top-gates. The distance between back- and top-gates is held fixed at $40 \mathrm{~nm}$, while the quantum well, of variable thickness, $z$, is centered $15 \mathrm{~nm}$ above the back-gate. 


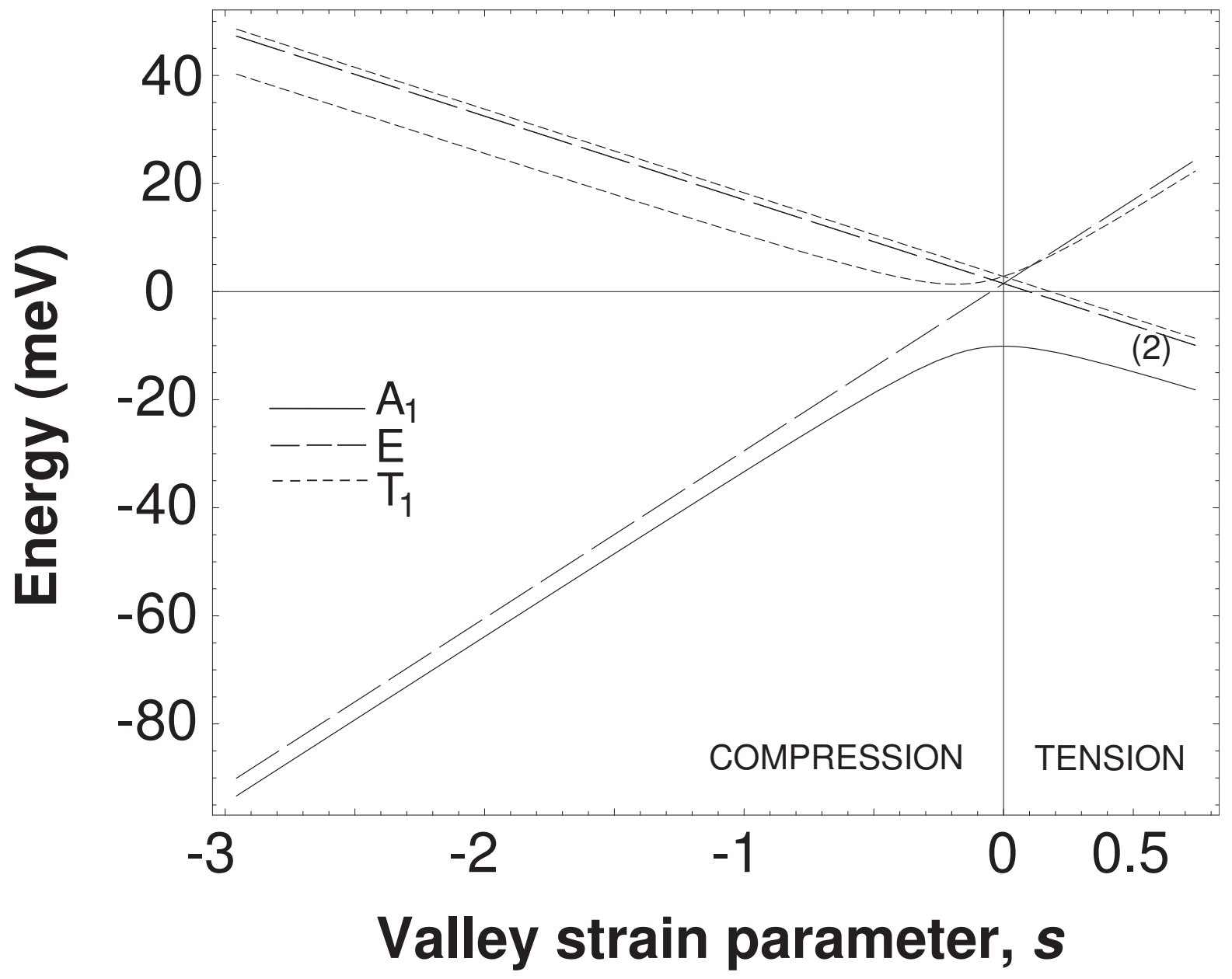

FIG. 3: Energy of the six 1s-like donor levels of an electron bound by a $\mathrm{P}$ impurity in silicon (with respect to the energy center of gravity) versus the strain parameter $s$ with an uniaxial stress applied in the [100] direction. (It is important to note for clarity that what we call compression relative to the [100] or growth direction is equivalent to tension in the plane of the quantum well. This label convention differs across fields.) For reference, $s=-3$ corresponds to the compressive (s negative) strain caused in a pure silicon layer by a $\mathrm{Si}_{0.8} \mathrm{Ge}_{0.2}$ sublayer. The energies are expressed in $\mathrm{eV}$ and the numbers in parenthesis indicate the degeneracy of the level. The $\mathrm{A}_{1}$ (ground state, solid line) and $\mathrm{T}_{1}$ level wave functions are mixed by strain (causing valley population intermixing) and the relaxation rate is proportional to $1 / \Delta E$. Thus, , with all else equal, an increase in strain causes the retaxation rate to decrease. For a detailed analysis see Wilson and Fehert, where $s$ here is their $s^{\prime}$ times 100 or Koiller et al. 24 whose notation is $s=100\left(6 \chi \Delta_{c} / \Xi_{c}\right)$. 


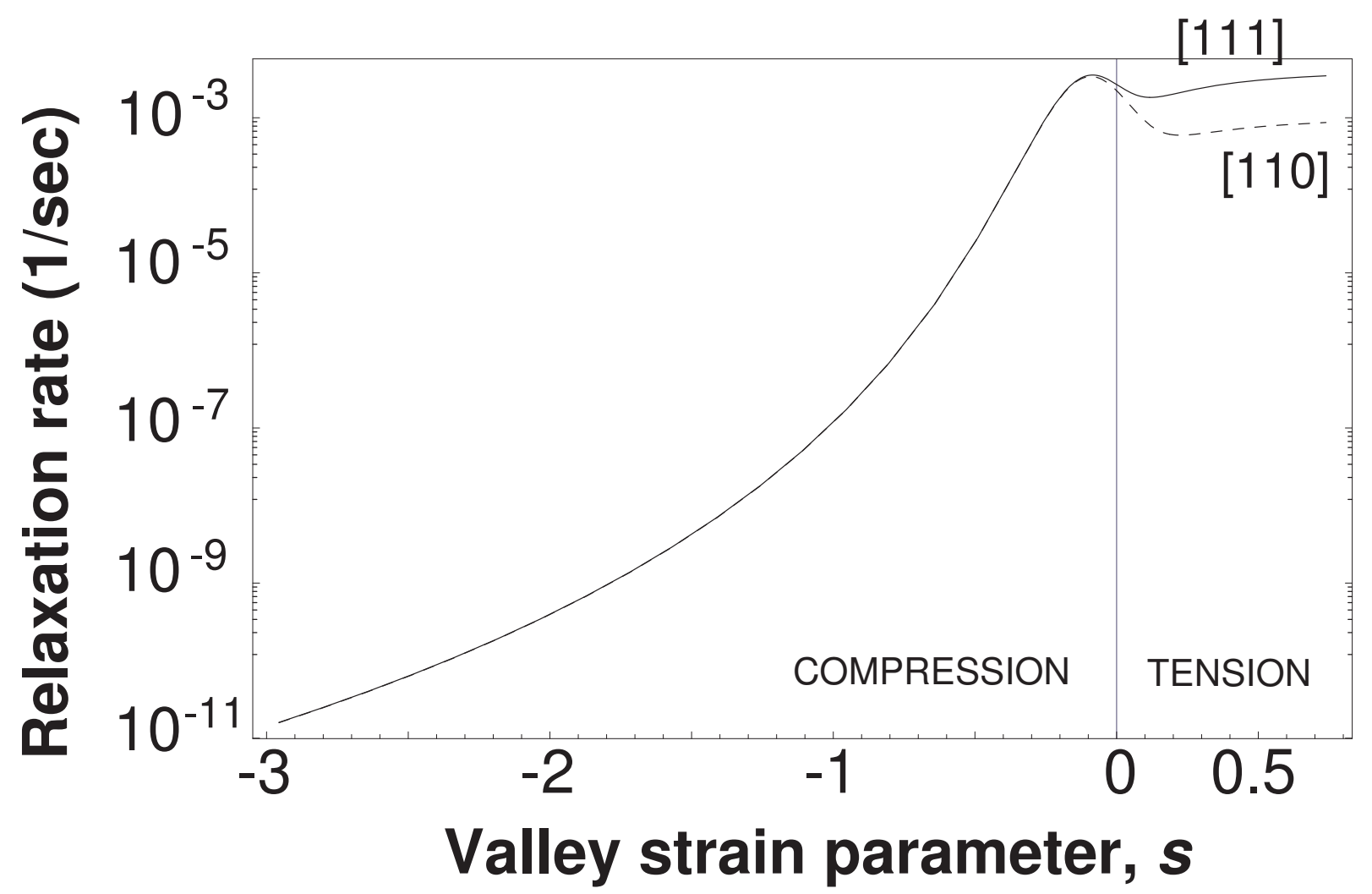

FIG. 4: Relaxation rates of a $\mathrm{P}$ impurity bound electron in [100] uniaxially strained silicon versus strain parameter $s$ for a temperature of 3 Kelvin and a magnetic field $H=H(\cos \theta \cos \phi, \cos \theta, \sin \theta)$ of 1 Tesla in the [111] $(\theta=\phi=\pi / 4)$ and [110] $(\theta=\pi / 4, \phi=0)$ directions respectively. $s=-3$ corresponds to the strain caused in the pure silicon layer by a $\mathrm{Si}_{0.8} \mathrm{Ge}_{0.2}$ sublayer. 

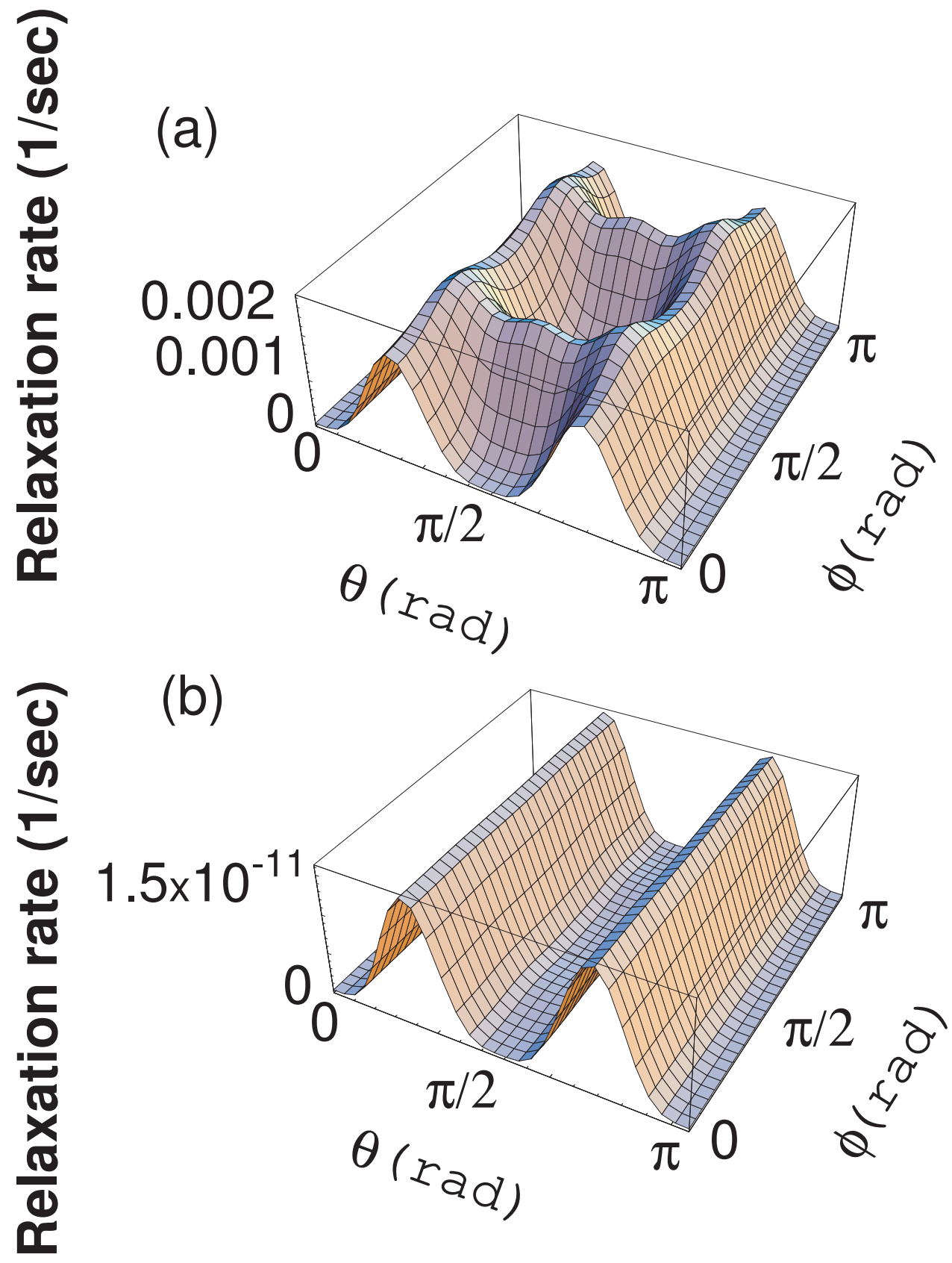

FIG. 5: Dependence of the relaxation rate on magnetic field direction with $H=H(\cos \theta \cos \phi, \cos \theta$, $\sin \theta)$ for (a) unstrained Si and (b) compressively strained $\mathrm{Si}$ (corresponding to a $\mathrm{Si}_{0.8} \mathrm{Ge}_{0.2}$ sublayer). The magnetic field is set at $1 \mathrm{~T}$ and the temperature is $3 \mathrm{~K}$. 


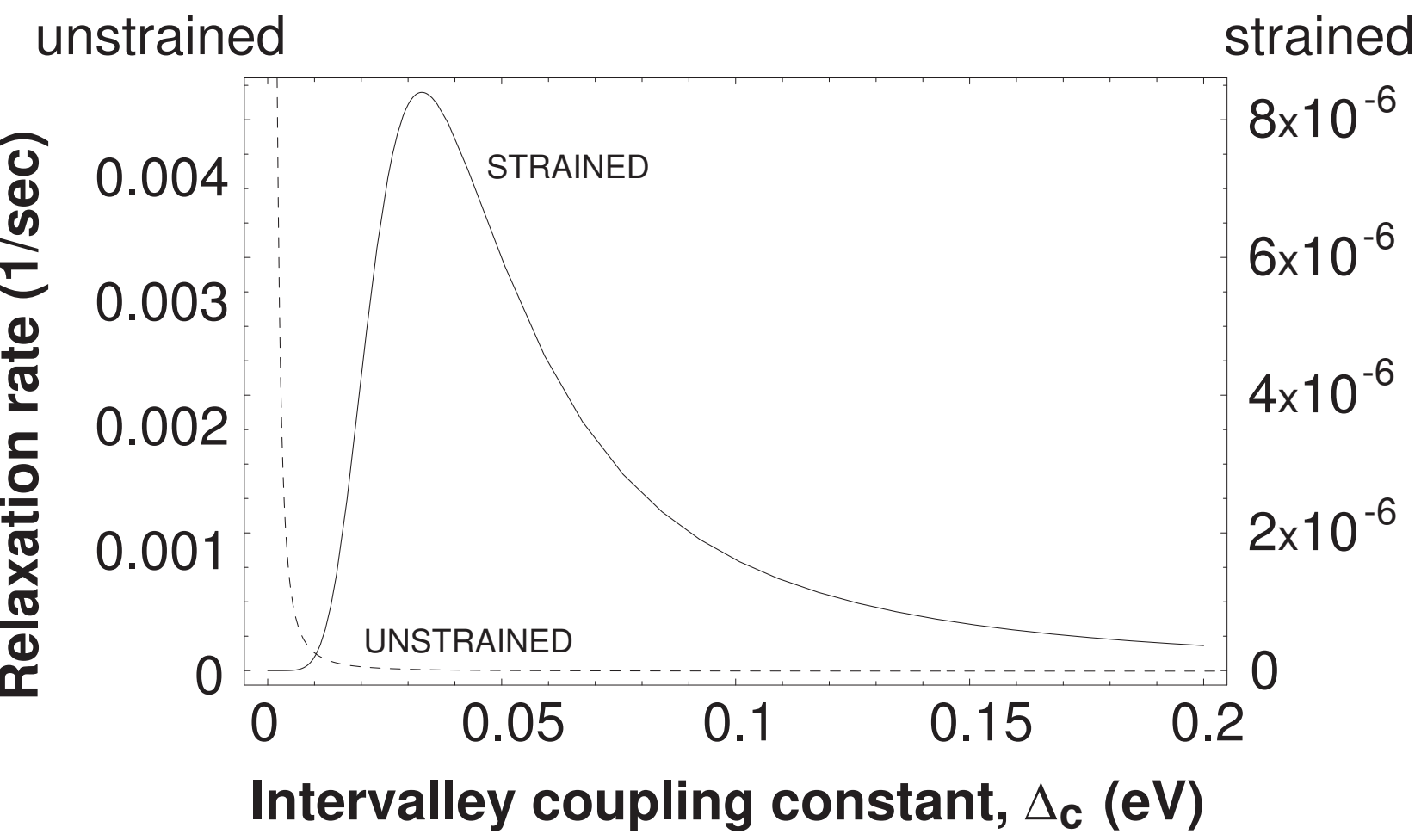

FIG. 6: Relaxation rate of a bound electron in silicon. The solid line is for fixed [100] uniaxial strain $s=-3$, corresponding to the strain caused in the pure silicon layer by a $\mathrm{Si}_{0.8} \mathrm{Ge}_{0.2}$ sublayer. The dashed line is the unstrained case. The rate is plotted for varying the intervalley coupling constant $\Delta_{c} . \Delta_{c}$ corresponds to $1 / 6$ th the energy splitting between the singlet (ground state) and triplet (next excited state) valley energies in unstrained $S i$. As a result, it controls the mixing between the $\pm x$ and $\pm y$ valley wavefunctions with the $\pm z$ valley wavefunctions. The magnetic field is set at $1 \mathrm{~T}$ along the [111] direction and the temperature is $3 \mathrm{~K}$. 

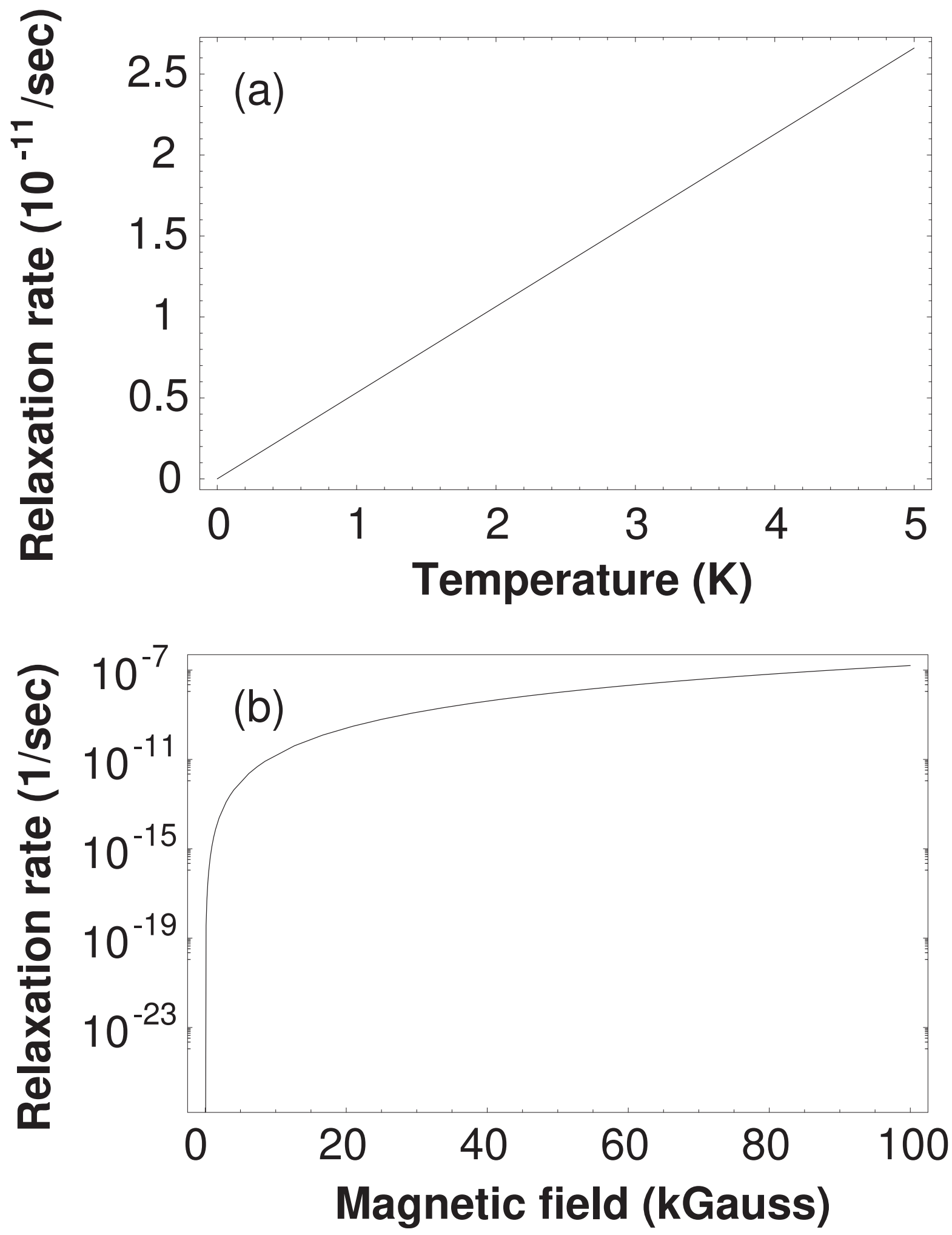

FIG. 7: Relaxation rates of a $\mathrm{P}$ impurity bound electron in [100] uniaxially strained silicon ( $s=-3$, corresponding to the strain caused in the pure silicon layer by a $\mathrm{Si}_{0.8} \mathrm{Ge}_{0.2}$ sublayer) versus (a) temperature with the magnetic field set at $1 \mathrm{~T}$ and (b) magnetic field strength with the temperature set at $3 \mathrm{~K}$. 


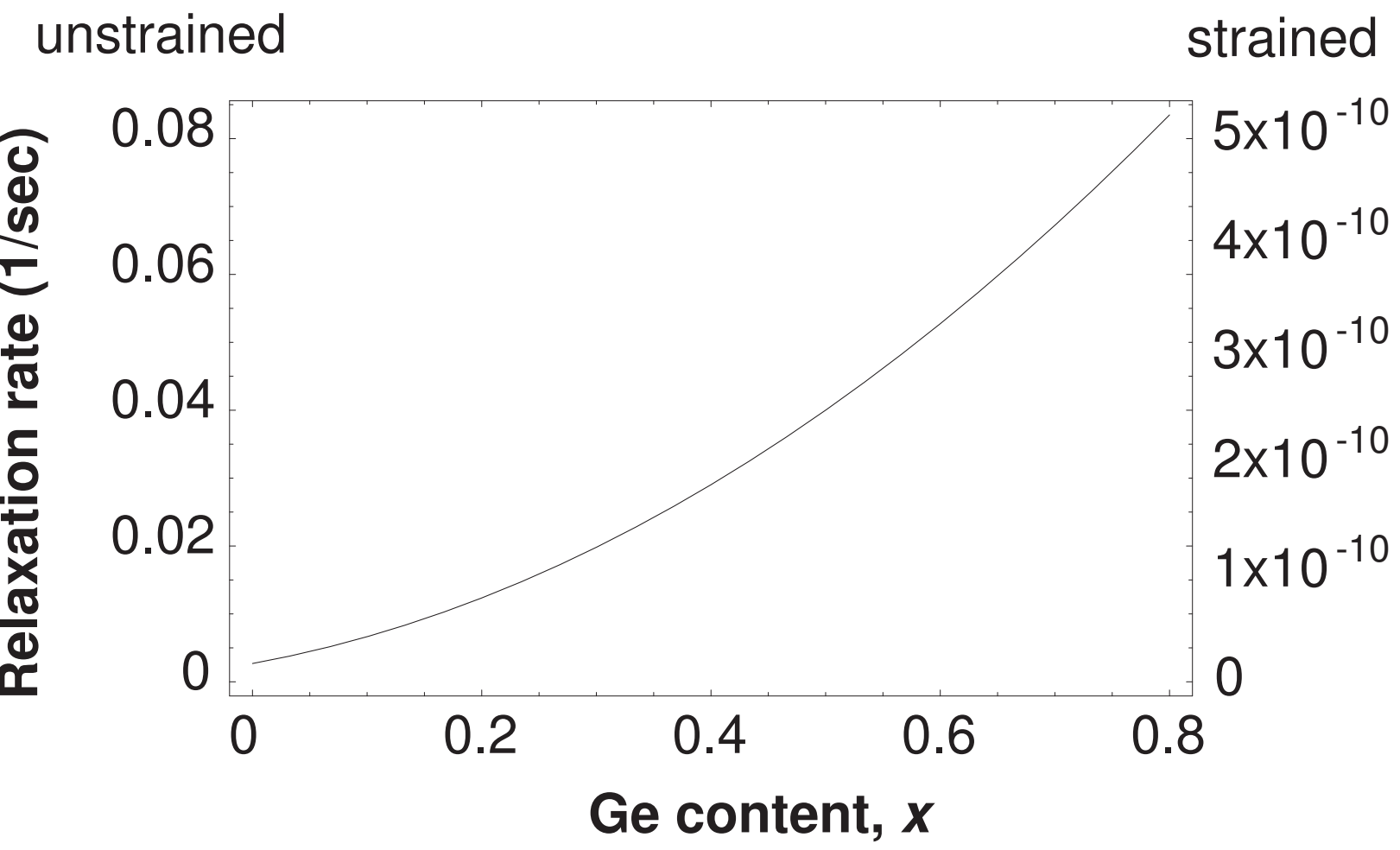

FIG. 8: Relaxation rate of a P impurity bound electron in [100] uniaxially strained silicon versus concentration of germanium within unstrained (left vertical axis) and compressively strained (right vertical axis, corresponding to a $\mathrm{Si}_{0.8} \mathrm{Ge}_{0.2}$ sublayer) pure silicon. The magnetic field is set at $1 \mathrm{~T}$ along the [111] direction and the temperature is $3 \mathrm{~K}$. Here we assume that the addition of germanium does not affect the strain within the bulk and only acts to increase the $g$-factor and spin orbit coupling. We expect this approximation to hold for small concentrations of germanium and to break down with increasing concentration as the conduction band valley minima switch from the $\mathrm{X}$ type of silicon to the L type of germanium. 